\title{
Caracterización del manejo en el ordeño de sistemas doble propósito del distrito de Los Santos ${ }^{\mathrm{a}}$.
}

\author{
Leonel Ríos ${ }^{1, *}$, Jaime Espinosa ${ }^{2,3}$, Jessica Hassan ${ }^{2}$. \\ ${ }^{1}$ Ingeniero en Producción Animal, Egresado de la Universidad Católica Santa María La Antigua (USMA), \\ Panamá, República de Panamá. \\ ${ }^{2}$ Centro de Investigación Agropecuaria de Azuero (CIAA), Instituto de Investigación Agropecuaria de \\ Panamá (IDIAP), Panamá, República de Panamá. \\ ${ }^{3}$ Profesor, Sede USMA Azuero, Universidad Católica Santa María La Antigua (USMA), Panamá, República de \\ Panamá.
}

*Autor para correspondencia. Email: riosleonel17@hotmail.com

Recibido: 10 de agosto de 2015

Aceptado: 14 de agosto de 2015

\begin{abstract}
The aim of the study was to characterize the milking management in dual-purpose farms in the district of Los Santos. Information from a probabilistic sample of 46 producers was gathered through semistructured interviews and direct observation, during the period from March to June 2014. The information comprised socio-economic aspects, characteristics of farms and milking management. Data was analyzed using descriptive statistics. Results show that $72 \%$ of farmers do not wash the udder of the cow at milking time. Most support their cows with calves, while $7 \%$ used injection (oxytocin) for support. $89 \%$ dry the udder at milking time, but with inappropriate practices such as drying of the teats with the cow's tail. The infrastructure for milking consists of a galley with floor for 35\% of producers, a galley without floor for 33\% of them, and 33\% milked in the corral. $80 \%$ sanitize utensils used after milking (sewers, jugs, buckets, liners and wipes). $96 \%$ clean the jugs of milk every day. 35\% reported having mastitis problems, mainly related to mechanized milking. We conclude that the producers showed inadequate management practices in both udder hygiene and the type of infrastructure, a situation that could compromise product quality and productivity of the herd.
\end{abstract}

Keywords: Dairy, Dual-purpose farm, Hygiene, Udder, Mastitis.

a Trabajo de investigación de tesis de Licenciatura en Ingeniería en Producción Animal de la USMA. Ríos L. 2015. 
Invest. pens. crit.

Vol. 3, No. 2, mayo-agosto 2015

pp. $5-19$

\section{Resumen}

El objetivo del estudio fue caracterizar el manejo del ordeño en fincas doble propósito del distrito de Los Santos. Se recolectó información a partir de una muestra probabilística de 46 productores, a través de encuestas semi-estructuradas y observación directa, durante el periodo marzo-junio 2014. La información comprendió aspectos socio-económicos, características de las fincas y del manejo al ordeño. Los datos se analizaron empleando estadísticas descriptivas. Se determinó que $72 \%$ de los productores no lavan la ubre de la vaca al momento del ordeño. La mayoría apoya a sus vacas con ternero, un 7\% utilizan inyección (oxitocina) para el apoyo. El 89\% realizan el secado de la ubre al momento del ordeño, pero con prácticas inadecuadas, como el secado de los pezones con el rabo de la vaca. La infraestructura para el ordeño consiste en galera con piso para el 35\% de los productores, 33\% disponen de galera pero sin piso, y 33\% ordeñan en el corral. El 80\% realiza la higiene de utensilios utilizados después del ordeño (coladeras, garrafones, cubos, pezoneras y paños de limpieza). El 96\% limpian los garrafones de leche todos los días. El 35\% indicaron tener problemas de mastitis, relacionada principalmente al ordeño mecanizado. Se concluye que los productores mostraron inadecuadas prácticas de manejo tanto en la higiene de la ubre como en el tipo de infraestructura, situación que puede llegar a comprometer la calidad del producto y la productividad de su hato.

Palabras clave: Lechería, Doble propósito, Higiene, Ubre, Mastitis.

\section{Introducción}

La leche de vaca es uno de los alimentos de mayor importancia mundial, de donde la población obtiene proteína diaria requerida. Algunos investigadores, han estimado que para el año 2050, se dará un incremento en la producción de leche y carne, superando más del 50\% de los niveles actuales de producción (Steinfeld et al. 2009). En Panamá, la producción de leche durante los últimos 15 años ha mostrado una tendencia hacía el aumento, consolidándose una tasa de crecimiento del $21 \%$ durante el periodo 2000-2014 (Figura 1).

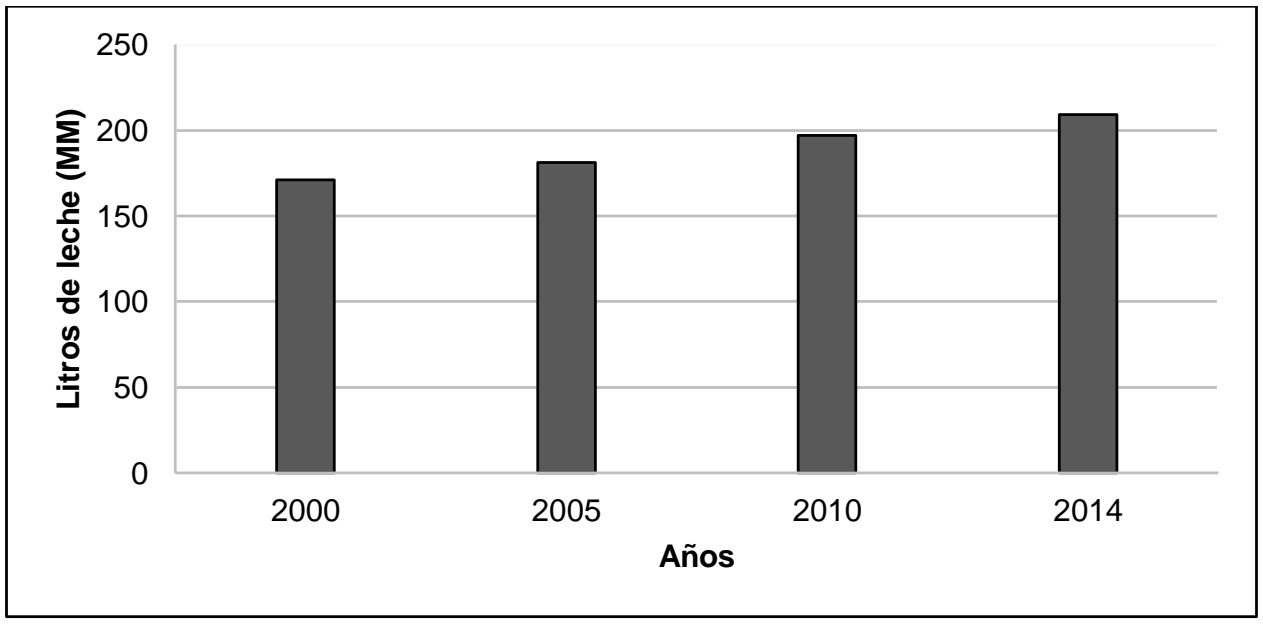

Figura 1. Producción de leche de vaca en Panamá, periodo 2000-2014. Elaborado por los autores a partir de datos del Instituto Nacional de Estadística y Censo (INEC, 2015). 
A nivel nacional se han identificado 5,338 explotaciones lecheras, principalmente el tipo de leche producida es Grado C o leche industrial. La lechería se ha considerado por años como una actividad tradicional de subsistencia, donde los productores, en su mayoría son pequeños, trabajan con los requerimientos básicos de producción, sin optimizar sus recursos y hacer más eficientes dichos procesos. La provincia de Los Santos ocupa el segundo lugar en número de explotaciones y litros de leche producidos en el país (Figura 2). Las explotaciones se caracterizan por desarrollarse en fincas pequeñas, con ordeño de tipo manual $(93 \%)$, y en muchos casos ser la principal fuente de ingreso para las familias rurales.

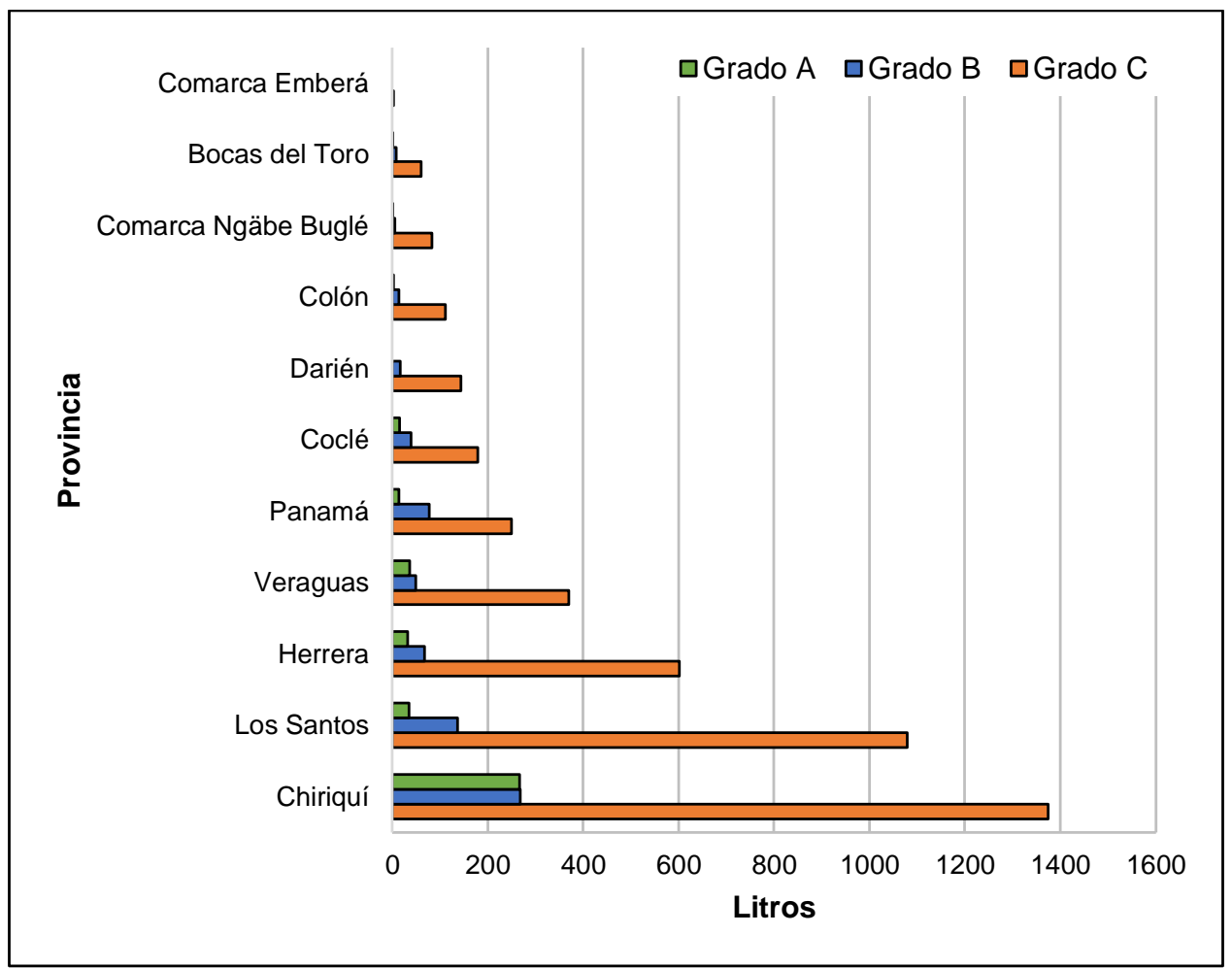

Figura 2. Tipo de leche producida (litros) según provincia y comarca indígena, semana del 17 al

23 de abril de 2011. Elaborado por los autores a partir de datos del INEC (2012).

Actualmente, la calidad de los productos lácteos se ve muy comprometida, tanto en las fincas como en las empresas colectoras y procesadoras. Una realidad muy marcada es que a nivel de fincas, los productores no toman importancia de las implicaciones que tiene el manejo durante el ordeño sobre la calidad del producto que envían a las empresas procesadoras. Generalmente, la información bibliográfica y estadística disponible, no es suficiente para desagregar las características que componen la población de explotaciones doble propósito. Esta situación, justifica la necesidad de realizar estudios de caracterización, que permitan conocer el manejo característico en el ordeño, así como los principales factores limitantes de la productividad en esos sistemas. 
Invest. pens. crit.

Vol. 3, No. 2, mayo-agosto 2015

pp. 5-19

\section{Materiales y métodos}

\section{Contexto.}

La investigación se desarrolló en el distrito de Los Santos, perteneciente a la provincia de Los Santos, ubicada en la zona oriental de la península de Azuero (Figura 3). El régimen de lluvias va de mayo a diciembre, con una precipitación media anual de 1,000 a 1,600 milímetros. La elevación varía desde los manglares a nivel del mar hasta los $400 \mathrm{~m}$ de altura, sin embargo, la mayoría de las fincas lecheras se encuentran por debajo de los 100 msnm. La zona de vida, según Holdridge, es bosque seco tropical (Jones 1982).

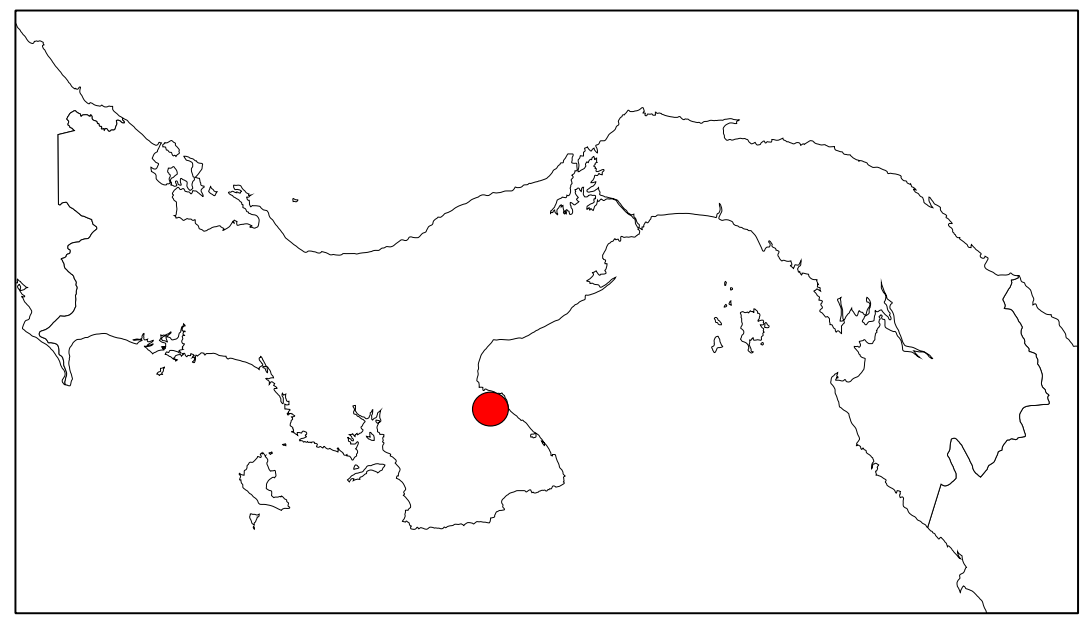

Figura 3. Ubicación del área de estudio.

Muestra.

Se consideró como universo a los 399 productores de leche del sistema doble propósito, reportado por el Instituto de Estadística y Censo de la Contraloría General de la República de Panamá (INEC, 2012).

Con base en la fórmula de cálculo de tamaño muestral para poblaciones finitas (Münch, citado por Pedroza, 2007), se determinó que la muestra consistiría de 46 productores de leche, para realizar estimaciones al 95\% de confianza, con un error estimado de $13 \%$ y una probabilidad esperada de $50 \%$. La fracción de muestreo $\mathrm{n} / \mathrm{N}$ representó aproximadamente un $12 \%$ del total de la población estudiada.

Generalmente el error de estimación aceptable para encuestas oscila desde un 2\% a 10\% (Pedroza, 2007). En nuestro caso, usamos un 13\% debido a limitaciones de presupuesto y logística para realizar las encuestas, así como para visitar las fincas de los productores para complementar con la observación directa en campo.

La muestra se seleccionó a partir del marco muestral de 399 productores de leche del sistema doble propósito. Esta lista incluyó a todos los productores de leche del sistema doble propósito que realizan ordeño de manera manual y mecanizada en el distrito de Los Santos. La selección de la muestra se 
realizó de manera aleatoria y proporcionalmente a la cantidad de productores existentes en cada corregimiento.

Tabla 1.

Composición del muestreo de los productores de leche.

\begin{tabular}{lccc}
\hline \multicolumn{1}{c}{ Corregimiento } & $\mathbf{N}$ & "n" propuesto & "n" logrado \\
\hline La Villa & 52 & 6 & 6 \\
El Guácimo & 26 & 3 & 2 \\
La Colorada & 28 & 3 & 4 \\
La Espigadilla & 32 & 4 & 1 \\
Las Cruces & 20 & 2 & 3 \\
Los Ángeles & 21 & 3 & 3 \\
Los Olivos & 41 & 5 & 6 \\
Llano Largo & 18 & 2 & 0 \\
Sabana grande & 25 & 3 & 4 \\
Santa Ana & 57 & 7 & 6 \\
Tres Quebradas & 22 & 3 & 3 \\
Agua Buena & 10 & 1 & 2 \\
Villa Lourdes & 22 & 3 & 3 \\
Las Guabas & 25 & 3 & $\mathbf{n}=\mathbf{4 6}$ \\
\hline \multicolumn{1}{c}{ Total } & $\mathbf{N = 3 9 9}$ & $\mathbf{n = 4 8}$ &
\end{tabular}

$\mathrm{Al}$ momento de realizar el trabajo de campo, se encontraron algunas restricciones respecto al muestreo. En total se obtuvieron 46 encuestas a nivel de los productores (Tabla 1), faltó entrevistar a 4 productores para completar la n propuesta. Para el caso de los corregimientos de Llano Largo y La Espigadilla, no se pudo encontrar a los productores debido a que sus ubicaciones no fueron encontradas o estaban incorrectas. También, algunos productores estaban ausentes a la hora de la visita, por lo que, en ocasiones, hubo que reprogramar las visitas.

\section{Materiales.}

Para la recolección de datos se empleó la consulta de información secundaria y la obtención de información primaria a partir de las encuestas a los productores, complementada con la observación directa en campo.

Se diseñó un formulario de encuesta semi-estructurada, con preguntas abiertas y cerradas, siendo estas últimas, en algunos casos, dicotómicas y, en otros, de selección múltiple. El formulario de la encuesta para la caracterización comprendió aspectos socio-económicos, características de las fincas, aspectos de manejo del ordeño y los problemas del sistema.

Previamente a la realización de las encuestas, esta fue discutida con los encuestadores y se validó a través de encuestas piloto. 
Invest. pens. crit.

Vol. 3, No. 2, mayo-agosto 2015

pp. 5-19

\section{Procedimiento.}

La investigación es de tipo descriptiva con diseño transversal. Se utilizó la metodología propuesta por Palencia (1993) para realizar una caracterización general de los sistemas de producción, haciéndose énfasis en los aspectos del manejo en el ordeño. Las variables de información a recopilar correspondieron a los siguientes temas o descriptores:

- Características personales del productor: edad, sexo, procedencia, educación, experiencia en el manejo de la finca y el número de años que ha tenido la finca.

- El uso de la tierra: La escala de las actividades pecuarias.

- El nivel tecnológico en los diferentes sistemas pecuarios: la producción de leche, la carga animal, entre otros.

- Aspectos del manejo ex-ante y ex-post el ordeño.

- El destino de la producción (autoconsumo o producción para el mercado), así como el mercado de los productos destinados para la venta.

- Los problemas más relevantes en los sistemas pecuarios.

- Las opiniones del productor sobre una serie de elementos claves en el proceso de desarrollo agropecuario, como son: a) funcionamiento de las instituciones en la zona, b) funcionamiento de las organizaciones agrícolas, c) principales problemas que afectan a la producción y las condiciones de vida en general, y d) percepciones del productor referente al futuro de la finca, de los hijos y de la zona en general.

Los datos recolectados fueron tabulados en hojas de cálculo de MS Excel® para la elaboración de una base de datos. Los datos fueron analizados estadísticamente con el programa InfoStat ${ }^{\circledR}$.

El análisis estadístico de los datos fue de tipo descriptivo e incluyó:

- Análisis de tablas y gráficos de frecuencias

- Medidas de tendencia central (Media, Mediana, Percentiles)

- Medidas de dispersión (Desviación Estándar, Coeficiente de Variación, Rango)

- Análisis de tablas de contingencia para variables cualitativas

- Análisis de correlación de Pearson

\section{Resultados}

Características socioeconómicas.

Los productores de leche de los sistemas doble propósito, en el distrito de Los Santos, poseen una edad mediana de 62 años. Respecto a los años de experiencia dedicados al sistema doble propósito, tienen una mediana de 40 años de experiencia, esta variable estuvo altamente correlacionada $(\mathrm{r}=0.80)$ con la variable edad de los productores. 
De los ganaderos encuestados, el 59\% indicó contar con algún familiar que colabora en la finca, principalmente son hijos, sobrinos o nietos. La mayoría, el 85\% de los productores emplean jornal eventual; la mano de obra en la finca es prácticamente familiar. El 35\% de estos ganaderos trabajan fuera de su finca como fuente de más ingresos para el hogar. En su mayoría los productores son alfabetos, principalmente con niveles de escolaridad secundaria (41\%), primaria (30\%), y niveles técnicos (22\%).

\section{Características del sistema doble propósito}

La carga animal promedio de los sistemas doble propósito del distrito de Los Santos fue de 1.5 animales $\mathrm{ha}^{-1}$. La mediana del número de vacas de ordeño en la época lluviosa fue de 18 vacas, y para la época seca fue de 13 vacas. La producción de leche en litros tuvo para la época lluviosa una mediana de 6 litros vaca ${ }^{-1}$, y para la época seca de 3 litros vaca ${ }^{-1}$.

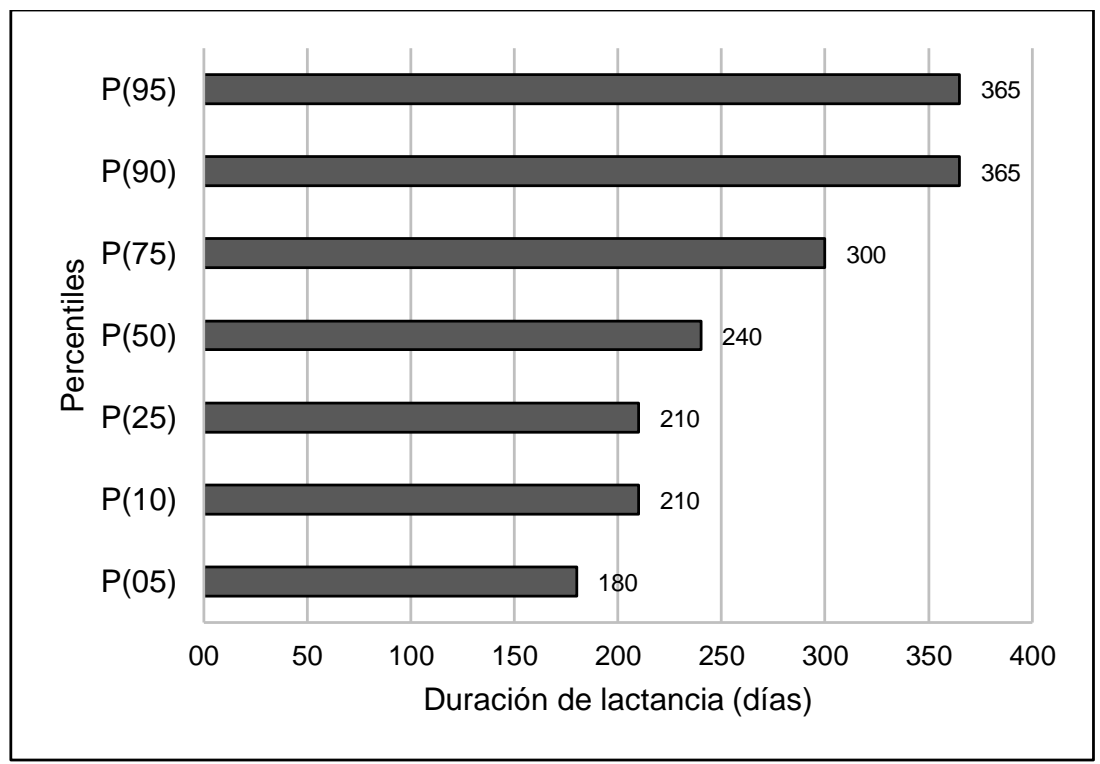

Figura 4. Duración de la lactancia, distrito de Los Santos, 2014.

En la Figura 4, se muestran los datos de la duración de la lactancia de los sistemas doble propósito. El promedio de duración de la lactancia fue de 256 días, con una dispersión de 59 días con respecto al promedio y un coeficiente de variación de $23 \%$.

\section{Características en el manejo del ordeño}

El tipo de apoyo a la hora del ordeño es principalmente con ternero (Figura 5). Los que realizan ordeño manual apoyan con terneros principalmente, los que hacen ordeño mecanizado, optan por inyecciones, concentrado y en menor frecuencia con los terneros. 
Invest. pens. crit.

Vol. 3, No. 2, mayo-agosto 2015

pp. 5-19

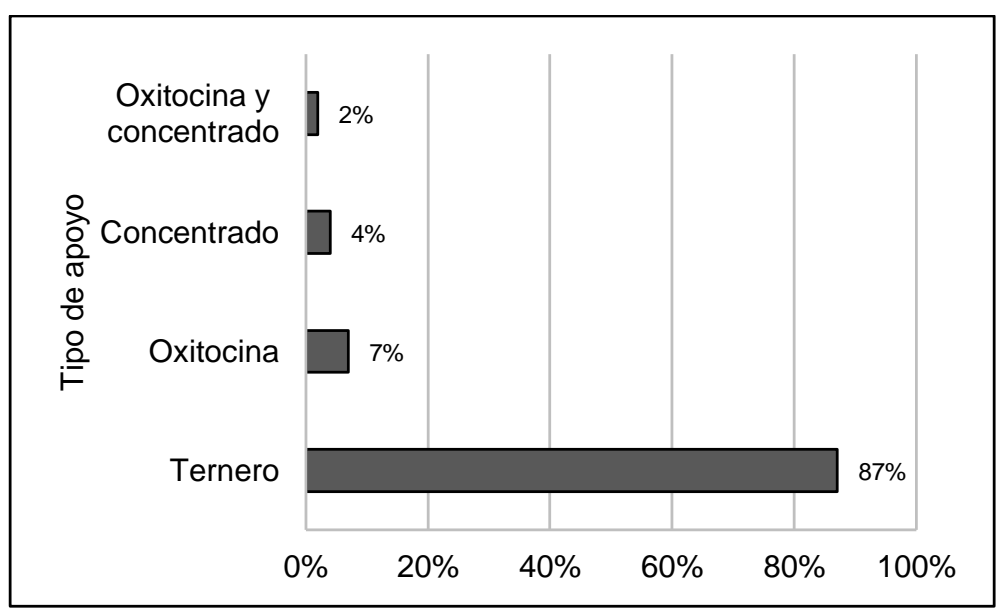

Figura 5. Tipos de apoyo utilizados por los productores del distrito de Los Santos, 2014.

En cuanto a la infraestructura para el ordeño una tercera parte $(35 \%)$ de los productores tiene galera con piso, otros $(33 \%)$ disponen de galera pero sin piso y otros $(33 \%)$ ordeñan en área libre donde solo tiene el corral. Son pocos los productores que tienen alguna fuente de agua potable o agua de pozo en su corral. Respecto a la higiene de la ubre al ordeño, la mayoría de los productores encuestados indicó que nunca lavan la ubre de la vaca al momento del ordeño (Figura 6).

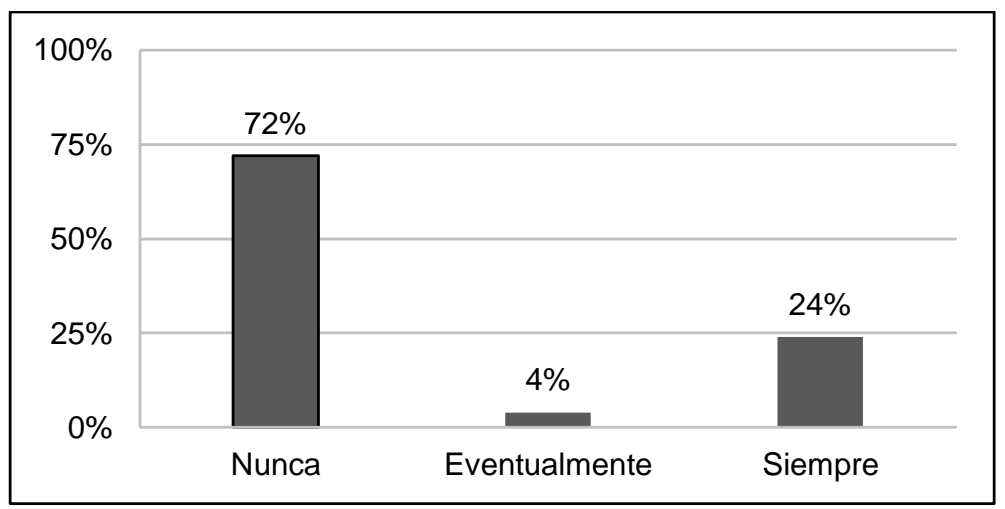

Figura 6. Frecuencia del lavado de la ubre al momento del ordeño por los productores en el distrito de Los Santos, 2014.

La mayoría de los productores indicó realizar el secado de la ubre al momento del ordeño. Sin embargo, más de la mitad de los productores secan los pezones al momento del ordeño con el rabo de la vaca y el resto de los productores con toallas de tela por grupo de animales (Figura 7).

Relativo a la limpieza del equipo de ordeño, todos los productores que ordeñan de forma mecanizada $(7 / 46=15 \%)$ indicaron que realizan la limpieza de las pezoneras todos los días. Para limpiar las pezoneras los principales productos que utilizan frecuentemente son jabón, cloro y yodo. 


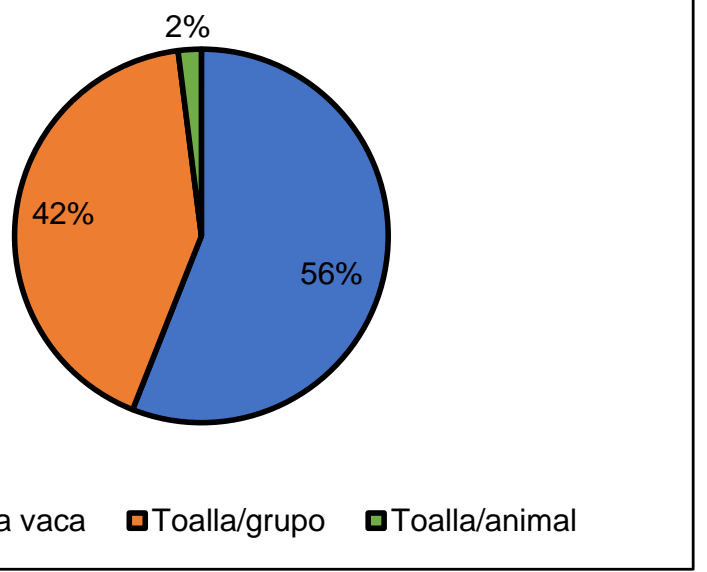

Figura 7. Frecuencia de las prácticas del secado de la ubre al ordeño por los productores en el distrito de Los Santos, 2014.

De los productores encuestados el 35\% (16/46) indicó tener problemas de mastitis con sus vacas, el análisis de tabla de contingencia y la prueba de Chi Cuadrado Pearson $(\mathrm{p}<.05)$ mostraron que la presencia de mastitis está significativamente asociada a los productores que ordeñan mecanizado (Tabla 2).

Tabla 2.

Presencia de mastitis según el tipo de ordeño en el distrito de Los Santos.

\begin{tabular}{cccc}
\hline Mastitis & Manual & Mecanizado & Total \\
\hline No & $74 \%$ & $14 \%$ & $65 \%$ \\
Sí & $26 \%$ & $86 \%$ & $35 \%$ \\
\hline Total & $\mathbf{1 0 0} \%$ & $\mathbf{1 0 0 \%}$ & $\mathbf{1 0 0} \%$ \\
\hline Chi Cuadrado Pearson & $=9.44$ & $\mathrm{p}<.05$ &
\end{tabular}

Cerca de la totalidad de los productores (96\%) indicaron que limpian los garrafones de leche. De estos productores la mayoría indicó que realiza la limpieza de los garrafones todos los días. En el caso de los dos productores (4\%) que no limpian los garrafones, estos comentaron que en la empresa los lavan y que cuando el garrafón llegaba a su residencia ya no limpiaban. En la Figura 8, se presenta la frecuencia de limpieza de garrafón.

Los productos que utilizan para la limpieza de los garrafones son principalmente clorox y jabón. La mayoría utiliza jabón en polvo, y son pocos los productores que utilizan jabón líquido y jabón especial para limpieza de utensilios de lechería.

Respecto a la limpieza de filtro o coladera el 65\% (30/46) de los productores indicaron que sí limpian sus coladeras. Un análisis de tablas de contingencia mostró que esta variable no está asociada ( $\mathrm{p}=.6517)$ a la limpieza de garrafones, por lo que, de los productores que lavan sus garrafones, existen tanto que limpian como que no limpian sus coladeras. La frecuencia de limpieza de la coladera según indicaron los productores es todos los días. Los productos utilizados para la limpieza son clorox y jabón. 
Invest. pens. crit.

Vol. 3, No. 2, mayo-agosto 2015

pp. 5-19

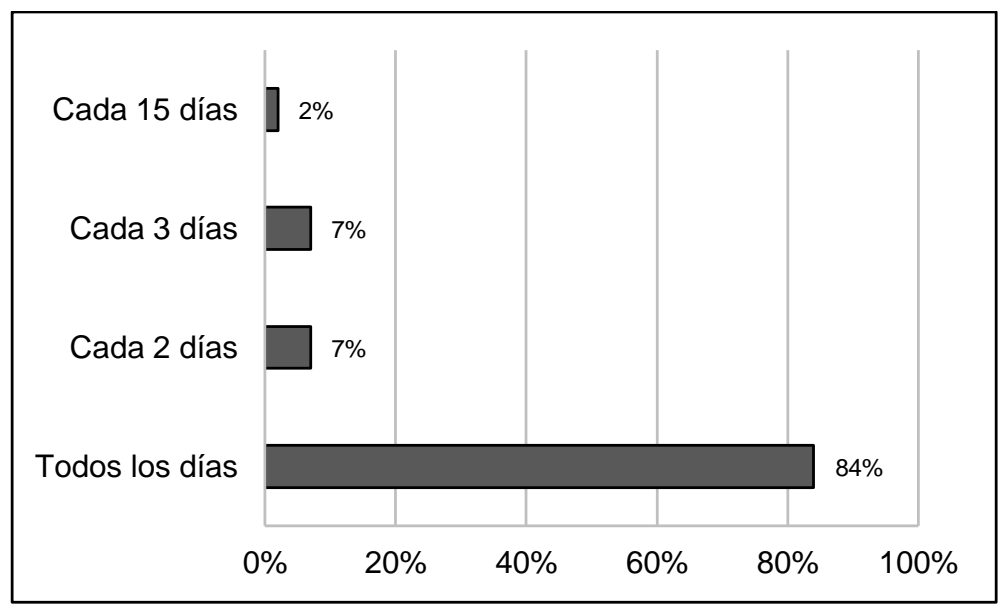

Figura 8. Frecuencia de la limpieza de garrafones de leche por los productores en el distrito de Los Santos, 2014.

El 89\% (41/46) de los productores encuestados indicaron que sí lavan sus cubos con una frecuencia diaria. Igualmente indicaron que los productos utilizados para la limpieza son clorox y jabón.

La hora de inicio del ordeño de los productores encuestados se concentra a las 5:00 am, con un 14\% de variación. La mediana de la hora en que finalizan los productores el ordeño es a las 6:30 am, con un 9\% de variación. La mediana de la hora en que la leche llega a la empresa es a las 8:00 am, con un 9\% de variación (Figura 9).

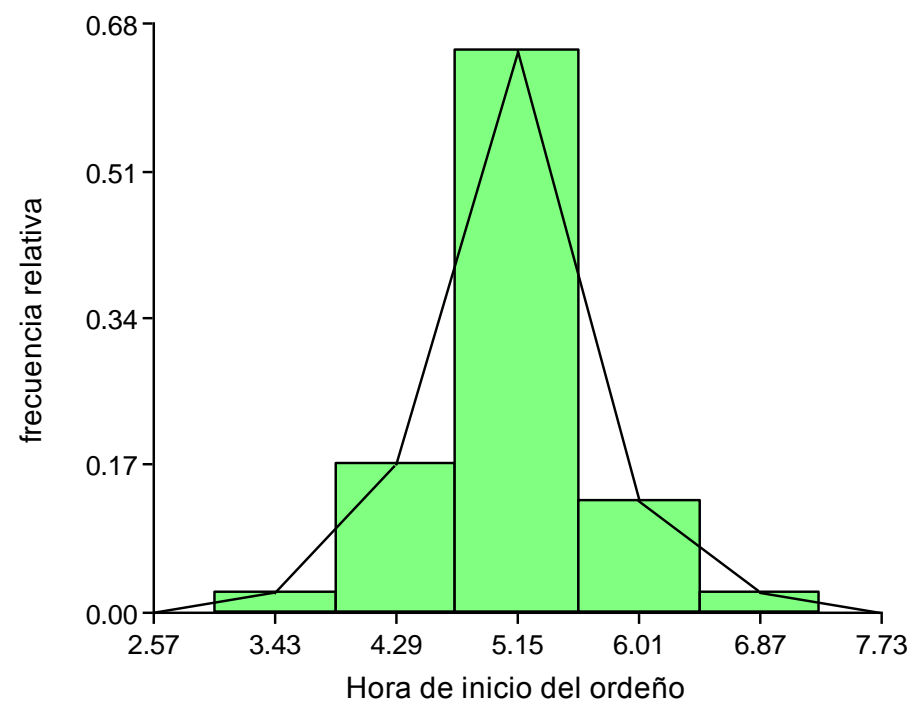

Figura 9. Histograma de la hora de inicio del ordeño por los productores en el distrito de Los Santos, 2014. 
Mercados de destino de la leche

Los productores encuestados reflejaron la participación de 8 mercados de destino de la leche. No obstante, la mitad del destino se concentró en las empresas Nestlé y Salvalac; el resto se concentró principalmente en Quesos Doña Mery, Estrella Azul y Prolacsa.

Según los datos del precio actual por litro de leche referido por los productores encuestados, la media y mediana es de USD $\$ 0.45$, con una variación de $13 \%$. El precio mínimo referido por los productores fue de USD $\$ 0.38$ y el precio máximo fue de USD $\$ 0.75$. Este último se considera un dato atípico ya que solo fue referido por uno de los productores, debido a que este productor produce su propio queso y establece su precio de venta.

\section{Perspectivas sobre el futuro}

Con respecto a las perspectivas que tienen los productores sobre el futuro de la actividad lechera en su entorno, estos emitieron sus consideraciones sobre algunas limitantes y necesidades actuales de la actividad.

El 67\% de los productores indicó que la actividad de ganadería de leche es su principal actividad económica de sustento para el hogar. Los productores le otorgaron a esta actividad un peso relativo promedio de $68 \%$ de aporte a sus ingresos totales del hogar.

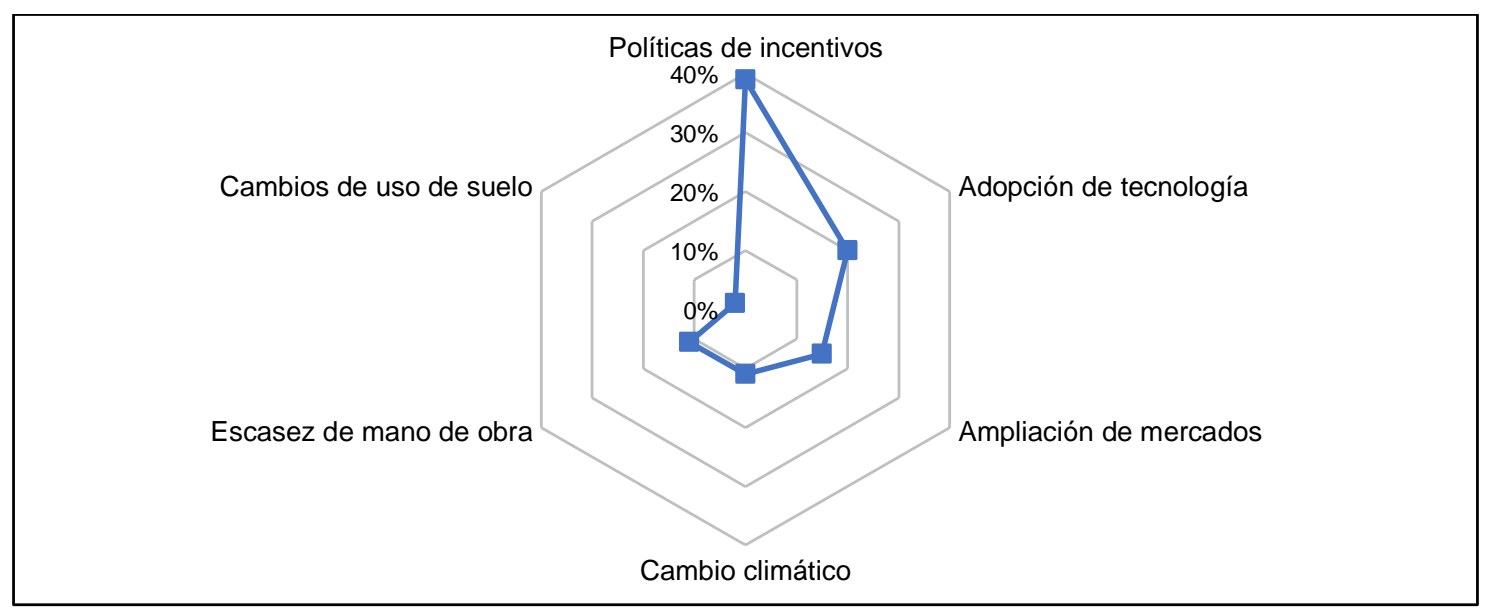

Figura 10. Perspectivas sobre limitantes y necesidades que inciden en el futuro de la actividad de los productores en el distrito de Los Santos, 2014.

Como puede apreciarse en la Figura 10, los productores opinaron que es necesario elaborar políticas de incentivos enmarcadas hacia la adopción de tecnologías, también consideran necesario para el futuro ampliar mercados, el cambio climático y la escasez de mano de obra son consideradas como serias limitantes para la actividad. 
Invest. pens. crit.

Vol. 3, No. 2, mayo-agosto 2015

pp. 5-19

\section{Discusión}

Estadísticamente, la población de productores del sistema doble propósito en el distrito de Los Santos, presentó un envejecimiento en cuanto a edad, situación que junto a los vastos años de experiencia, no refleja un cambio generacional. Según el Ministerio de Desarrollo Agropecuario (MIDA, 2007), es reconocido que la lechería panameña se caracteriza por desarrollarse en fincas pequeñas, con mano de obra familiar, siendo la principal fuente de ingresos para la familia rural, situación que concuerda con los resultados de este estudio.

El aumento de la producción de leche durante la época lluviosa (mayo a diciembre), se explica por el aumento en cantidad y calidad de los forrajes, lo cual permite los incrementos en la producción lechera; en esta época se produce cerca del 65\% del total de producción anual (MIDA 2007). En promedio la producción de leche diaria por vaca en la época lluviosa aquí encontrado, fue similar al promedio de 5.41 litros reportado en fincas doble propósito en Chiriquí, Panamá. En estudios de caracterización de la ganadería lechera en Yucatán, México y en otras regiones de Latinoamérica, los rangos de producción diaria por vaca oscilaron entre 2.8-6.5 litros (Guerra y González 1998; Osorio, Segura, Osorio y Marfil, 1999).

El ordeño único diario, realizado por la mayoría de los productores en este estudio, también es común en los sistemas de ganadería doble propósito orientados hacia la producción de leche en Centroamérica y Suramérica. Estudios en la zona oeste de Monagas, Venezuela mostraron que el 76\% de las fincas realizan un solo ordeño diario; en Veracruz, México el 94\% de las fincas lo realizan. El apoyo con ternero al momento del ordeño, al igual que en este estudio, también fue realizado en la mayoría de las fincas de la zona oeste de Monagas, Venezuela (97\%), no obstante, acostumbrar a las vacas al apoyo sin ternero, es una práctica que facilitaría la organización, rutina e higiene durante el ordeño, sin embargo, a un costo mayor (Alfaro, Díaz y Tirado, 1999; Vargas, Rubio y Corro, 2010). En la Tabla 3, se muestran características muy similares a las encontradas en este estudio, en fincas doble propósito de Venezuela.

Tabla 3.

Aspectos predominantes en fincas doble propósito de Venezuela.

\begin{tabular}{ccc}
\hline Variable & Descripción & $\begin{array}{c}\text { Frecuencia } \\
(\%)\end{array}$ \\
\hline Infraestructura para producción & Insuficientes & 48 \\
Nivel de equipamiento & Deficiente & 65 \\
Manejo sanitario & Deficiente & 79 \\
Ordeños/día & 1 & 87 \\
\hline
\end{tabular}

Fuente: Adaptado de "Caracterización estructural y funcional de fincas ganaderas de doble propósito en el municipio Páez del estado Apure, Venezuela”, L. Paez, T. Linares, W. Sayago y R. Pacheco, 2003, Zootecnia Tropical, 21(3), p. V.21, No.3 
En este estudio predominó el tipo de ordeño manual, situación que también se ha demostrado para el caso de los sistemas doble propósito en Venezuela (63\%) y México (82\%). El aspecto sanitario de higiene de la ubre antes del ordeño, probablemente el más relevante de los aspectos de manejo, fue bajo en Veracruz, México donde solo 18\% de los productores realizan la higiene de la ubre, variable que para el caso del distrito de los Santos (24\%) también consideramos baja.

La presencia de problemas de mastitis indicada mayormente por productores que realizan un ordeño mecanizado, demuestra que existe un problema de higiene, si bien, los productores mencionaron que realizan la limpieza de las pezoneras con frecuencia diaria.

\section{Conclusiones}

Comúnmente los ganaderos del sistema doble propósito en el distrito de Los Santos son mayores de 50 años, reflejándose un envejecimiento de la población productiva de estos sistemas, lo que pudiese influir en la capacidad de ser sujeto a créditos y adoptar nuevas tecnologías.

La ganadería es la principal fuente de ingreso de los hogares de los ganaderos encuestados, sin embargo, indicaron limitantes que les impide mejorar sus sistemas productivos, como la ausencia de políticas de incentivo, la escasez de mano de obra, la necesidad de nuevos mercados, facilidades en créditos y los efectos de la variabilidad climática.

En general existe poca higiene al momento del ordeño en relación a la limpieza de la ubre y una infraestructura inadecuada, situación que puede comprometer la calidad del producto. Sin embargo, los productores señalaron que sí realizan la higiene después del ordeño con el lavado de los utensilios que son utilizados durante el ordeño y el transporte a las plantas procesadoras.

\section{Referencias}

Alfaro, C., Díaz, V. C., y Tirado, H. (1999). Caracterización sanitaria de la ganadería doble propósito en el municipio Ezequiel Zamora del estado Monagas-Venezuela. Veterinaria Tropical, 24(2), 103-119.

Castro Ramírez, A. (2002). Ganadería de leche: Enfoque empresarial. Producción bovina (Tomo 1). San José, Costa Rica: Editorial Universidad Estatal a Distancia.

Centro Internacional de Mejoramiento de Maíz y Trigo [CIMMYT]. (1993). La adopción de tecnologías agrícolas: Guía para el diseño de encuestas. México, D.F.: Programa de economía del CIMMYT.

Guerra M. P. y González, A. (1998). Tipificación de fincas doble propósito en la provincia de Chiriquí, Panamá. Ciencia Agropecuaria, (9), 153-170.

Hernández Sampieri, R., Fernández Collado, C., y Baptista Lucio, P. (2010). Metodología de investigación

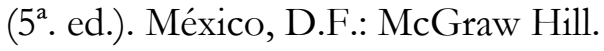


Invest. pens. crit.

Vol. 3, No. 2, mayo-agosto 2015

pp. 5-19

Instituto Nacional de Estadística y Censo [INEC]. (2012). VII Censo Nacional Agropecuario. Resultados Finales Básicos, 2011. Volumen 4. Características de la Actividad Pecuaria. Panamá: Autor. Disponible en http://www.contraloria.gob.pa/inec/

Instituto Nacional de Estadística y Censo [INEC]. 2015. Situación Pecuaria: Año 2014. Panamá: Autor. Disponible en http://www.contraloria.gob.pa/inec/

Jones, J. R. (1982). Diagnóstico socio-económico sobre el consumo y producción de leña en fincas pequeñas de la Peninsula de Azuero, Panamá. (Informe Técnico No. 32). Turialba, Costa Rica: Centro Agronómico Tropical de Investigación y Enseñanza.

Lagares B., P. y Puerto A, J. (2001). Población y muestra: Técnicas de muestreos. Management Mathematics for European Schools. Disponible en: http://optimierung.mathematik.uni-kl.de/mamaeusch/veroeffentlichungen/ver_texte/sampling_es.pdf

Martiz, G. y Vergara, L.K. (2004). Caracterización de la actividad ganadera en las subcuencas de Los Hules, Tinajones y Caño Quebrado. Panamá: Comisión Interinstitucional de la Cuenca Hidrográfica del Canal $(\mathrm{CICH})$.

Ministerio de Desarrollo Agropecuario [MIDA]. (2007). Plan estratégico para el desarrollo del Subsector Lechero, 2007-2013. Panamá: Autor. Disponible en http://www.mida.gob.pa/upload/documentos/plan-nacional-de-leche\%5B1\%5D.pdf

Osorio, M. M., Segura, J. C.; Osorio, D.A. y Marfil, A. A. (1999). Caracterización de la ganadería lechera del estado de Yucatán, México. Rev. Biomed. 10(4), 217-227.

Páez, L., Linares, T., Sayago, W. y Pacheco, R. (2003). Caracterización estructural y funcional de fincas ganaderas de doble propósito en el municipio Páez del estado Apure, Venezuela. Zootecnia Tropical, 21(3). 2015. Disponible http://www.scielo.org.ve/scielo.php?script=sci_arttext\&pid=S0798-72692003000300006

Palencia O., A. (1993). Caracterización de sistemas agrícolas para efectos de generación-transferencia de tecnología apropiada. Conceptos, terminología, estrategia y elementos para la caracterización (Documento técnico 3). San José, Costa Rica: Publicaciones PRIAG.

Pedroza, H. (2007). Enfoque integrado de investigación y extensión en sistemas agropecuarios: Enfoque IESA. Managua, Nicaragua: Instituto Interamericano de Cooperación para la Agricultura (IICA) / Instituto Nicaragüense de Tecnología Agropecuaria (INTA).

Steinfeld, H., Gerber, P., Wassenaar, T., Castel, V., Rosales, M. y De Haan, C. (2009). La larga sombra del ganado: Problemas ambientales y opciones. Roma, Italia: Organización de las Naciones Unidas para la Agricultura y la Alimentación (FAO). 
Invest. pens. crit.

Vol. 3, No. 2, mayo-agosto 2015

pp. 5-19

Vargas C., Y., Rubio G., I., Corro M., M. D. (2014). Caracterización del Manejo en Sistemas de Producción Bovina de Doble Propósito en el Municipio de Vega de Alatorre, Veracruz. Revista Entorno Ganadero. Año 9, No. 63. Disponible en:

http://bmeditores.mx/wp-content/uploads/2014/02/Ganadero63.pdf 